\title{
Disturbance Rejection for Biped Humanoids
}

\author{
Sang-Ho Hyon ${ }^{\dagger, \ddagger}$ and Gordon Cheng ${ }^{\dagger, \ddagger}$ \\ † Computational Brain Project, ICORP, JST \\ $\ddagger$ ATR, Computational Neuroscience Laboratories \\ Hikaridai 2-2-2, Souraku-gun, Kyoto 619-0288, Japan \\ \{sangho, gordon\} eatr.jp
}

\begin{abstract}
This paper proposes a simple passivity-based disturbance rejection scheme for force-controllable biped humanoids. The disturbance rejection by force control is useful not only for self-balance, but also for stable and safety physical interaction between human and humanoid robots. The core technique is passivity-based contact force control with gravitycompensation. This makes it easy to control the contact forces in a satisfactory dynamic range without canceling all non-linear terms. The disturbance rejection is located at the higher layer above the contact force controller. It is composed of three subcontrollers; 1) a balancing controller; 2) a stepping controller; and 3) the trigger. Numerical simulations and experiments evaluate the effectiveness of the proposed controller. Although the method is incomplete in the sense that the self-collision between the limbs is ignored, a preliminary experimental result on a real humanoid platform demonstrates that the proposed method can actually make the robot recover the balance under large unknown external perturbations.
\end{abstract}

Index Terms-Passivity, Disturbance rejection, Humanoid, Balance, Walking.

\section{INTRODUCTION}

Safety will be the most important factor for any robotic systems operating in the real world. Disturbance rejection serves as one of the safety requirements for full-sized humanoid robots - it prevents the robot from falling and injuring people and/or itself.

Several balancing compensators and compliance controllers have been developed for position-controlled biped humanoid robots [1][2][3][4]. Although these works and others have demonstrated the validity of $\mathrm{CoP}$-constrained compensations, there remain three unsolved problems from the viewpoint of force interaction. Firstly, the methods always require the measurement of the external forces. Secondly, the non-causality between the measured forces and to-be-modified joint trajectories results in time-delay. ${ }^{1}$ Thirdly, being more problematic, it is difficult, in principle, to assign the weight, required for inverse kinematics, properly to unknown disturbances.

A force-based walking controller was proposed in [7] and applied to a planar biped walking robot by a novel force-controllable actuator prototype. But the extension to multi-DOF robots with multi-contact points and multi-DOF systems with redundant joints has not been presented. On the

\footnotetext{
${ }^{1}$ In this case, a preview control is required [5]. See [6] for the frequency domain analysis. The external disturbance can be incorporated only if it is estimated by observation in advance.
}

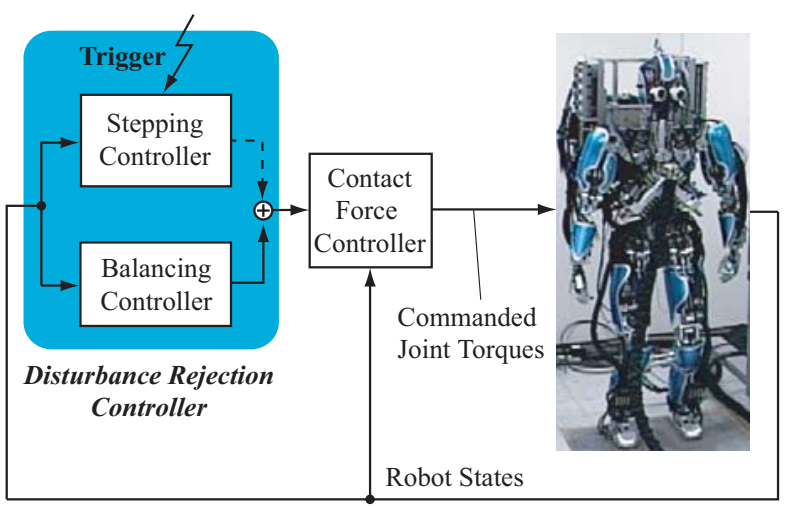

Fig. 1. Schematic overview of disturbance rejection control presented in this paper. The balancing and the stepping controller are simply combined. The stepping controller is triggered by the measured ground reaction force.

other hand, some recent works [8][9] extended a task-space control method [10] to full-body humanoid control. Although the methods achieved some desired task-space trajectories with given task priority and other constraints such as joint limits, they accompany with the exact cancellation of nonlinear dynamics and have not yet been applied to a real platforms.

In our study, we have been pursuing a passivity-based approach [11], where a passivity-based periodic motion control strategy proposed in [12][13] is generalized to multi-DOF humanoid robots. The underlying control policy is properly utilizing the non-linear dynamics of the system rather than cancelling them, as well as making the controller simple to implement and provable. The key element of the passivitybased approach is the invariance control [12]. This control transforms the original system into a new passified system by a feedback and control logic so that the resultant closed-loop solutions are bounded into some admissible region. It is an extension of passivation in non-linear control theory [14]. Once having obtained some recurrent orbits, new control inputs asymptotically stabilize them hierarchically.

The usefulness of this approach in the motion control of full-body, redundant humanoid robots has been demonstrated in [11] and [15]. They proposed a new passivitybased contact force control framework and demonstrated its effectiveness by various simulations including dynamic biped walking. In particular, [15] experimentally validated 
the method by showing our new biped humanoid platform (Fig. 1) can actually adapt to unknown external forces applied to arbitrary contact points.

As shown in [11] the novelty of our solution is the direct optimization of the contact forces through $\mathrm{CoP}$ in terms of the norm of the contact forces, which avoids the unnecessary internal forces between the contact points. In the contact control framework, gravity compensation plays a very important role since with an additional damping, we can approximately achieve desired contact forces. The gravity compensation is one of the most fundamental passivation controller because the robot becomes passive with respect to external forces and generalized velocities. Since the movement of the robot can be controlled by a very little amount of external forces, gravity compensation is clearly useful for physical interaction between human and humanoids [15].

This paper proposes a simple passivity-based disturbance rejection scheme in series with [11] and [15]. The controller is located at the higher layer above the contact force controller and includes three components: 1) balancing controller, 2) stepping controller, and 3) trigger, as depicted in Fig. 1. Section II describes briefly our passivity-based contact force control framework. Section III describes our disturbance rejection scheme and the simulation results. The simulation results are supplemented by the video clip in the conference DVD.Section IV demonstrates experimental results on our humanoid platform. They include balancing with unknown external forces and a preliminary result on disturbance rejection.

\section{PASSIVITY-BASED, FULL-BODY CONTACT FORCE CONTROL WITH GRAVITY COMPENSATION}

The most fundamental control issue for humanoid robots is the contact force control. This is because humanoid robots need to interact with the environment while keeping balance through these contact forces. Balancing, walking, and other interaction task can be easily derived when this contact force control framework is well established. This section briefly describes our contact force control framework. For the details see [11][15].

Consider a multi-DOF humanoid robot as shown in Fig. 2(a). Let $r_{C}=\left[x_{C}, y_{C}, z_{C}\right]^{T} \in \mathcal{R}^{3}$ be the position vector of CoM in the world coordinate frame $\Sigma_{W}$ and $q \in \mathcal{R}^{n}$ be the joint angles and attitude of the base. For our convenience, let's introduce a gross applied force, or ground applied force (GAF) $f_{P}=\left[\begin{array}{lll}f_{x P}, & f_{y P}, & f_{z P}\end{array}\right]^{T}$, defined as $f_{P}:=-f_{R}$, where $f_{R}$ is the ground reaction force (GRF). GAF represents the force that the robot applies to the environment. The control objective here is to bring $f_{P}$ to some desired value $\bar{f}_{P}$.

First, suppose the robot contact with the ground at a single point; $\mathrm{CoP}$ (center of pressure), which is represented by $r_{P}$ in Fig. 2. Later we will proceed to the multiple contact case. With the generalized coordinates $\hat{q}_{C}=\left[r_{C}, q\right]^{T} \in \mathcal{R}^{3+n}$, the exact nonlinear dynamics with the single contact constraint

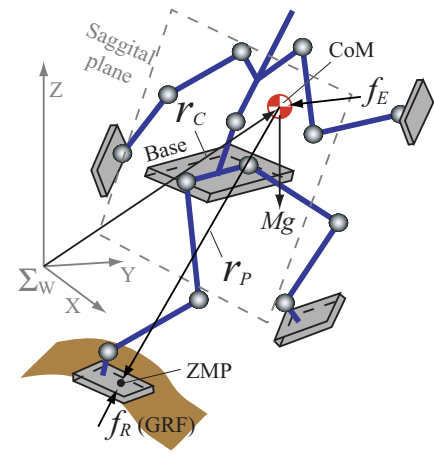

(a) CoM, CoP, and GRF

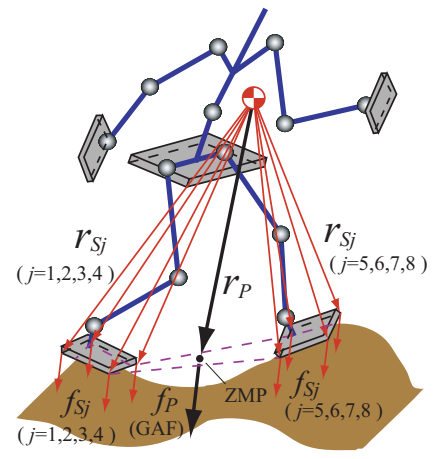

(b) Contact forces
Fig. 2. Definition of positions and forces. Each contact point $r_{S j}$ is assigned with the contact force $f_{S j}$. The sum of the contact forces $f_{S j}$ equals to GAF $f_{P}$. Note that $\operatorname{CoP} r_{P}$ always lies within the supporting convex hull composed of $r_{S j}$.

is described by

$$
\begin{aligned}
\underbrace{\left[\begin{array}{c|c}
M & 0 \\
\hline 0 & I(q)
\end{array}\right]}_{\hat{I}}\left[\begin{array}{c}
\ddot{r_{C}} \\
\hline \ddot{q}
\end{array}\right] & +\underbrace{\left[\frac{0}{C(q, \dot{q})}\right]}_{\hat{C}}+\underbrace{\left[\frac{M g}{G(q)}\right]}_{\hat{G}} \\
& =u-\hat{E}(q)^{T} f_{P}
\end{aligned}
$$

with $I(q)$ the inertia matrix, $C(q, \dot{q})$ the centrifugal and Coriolis term, $G(q)$ the gravity term, $u=[0, \tau]^{T} \in \mathcal{R}^{3+n}$, and $\hat{E}(q)$ the constraint Jacobian represented by

$$
\hat{E}(q)=\left[i d \mid J_{P}(q)\right],
$$

where $J_{P}(q) \in \mathcal{R}^{3 \times n}$ is the Jacobian from CoM to CoP (derivative of $r_{P}$ by $q$ ) and $i d$ is an identity matrix.

From (1) to (2) $f_{P}$ can be calculated as

$$
f_{P}=\left(\hat{E} \hat{I}^{-1} \hat{E}^{T}\right)^{-1}\left\{\gamma+\hat{E} \hat{I}^{-1}(u-\hat{C}-\hat{G})\right\}
$$

with $\gamma(q, \dot{q})=\frac{\partial}{\partial q}(\hat{E} \dot{q}) \dot{q}$. Therefore, for some desired GRF $\bar{f}_{P}$ we can calculate the corresponding joint torques $\tau$ by inverting (3). The torque precisely achieves $f_{P}=\bar{f}_{P}$ as long as the above model is correct. See [16] for more details on this type of exact GAF control for multi-link robots. Here, we want to avoid such an inverse dynamics because it becomes impractical for large DOF systems.

The followings are our practical solutions based on passivity. First, assume the motion is quasi-static. Since $\dot{q} \approx 0$ we can expect $\gamma \approx 0, C \approx 0$ (we cannot say $G \approx 0$ ). In this case, for some new force input $f_{u}=\left[f_{u x}, f_{u y}, f_{u z}\right]^{T} \in \mathcal{R}^{3}$, it is straightforward to see the joint torque

$$
\begin{aligned}
\tau & =J_{P}^{T}\left(f_{v}+M g\right) \\
f_{v} & =\left(J_{P} I^{-1} J_{P}^{T}\right)^{-1}\left(M^{-1}+J_{P} I J_{P}^{T}\right) f_{u}
\end{aligned}
$$

renders the closed-loop system satisfy

$$
f_{P} \approx f_{u}+M g
$$


This is the main idea of G-comp for controlling CoM [15]. A simpler formula is available by setting

$$
\tau=J_{P}^{T}\left(f_{u}+M g\right),
$$

instead of (4) and (5).

The advantage of using (7) is that we do not have to calculate $\gamma$ and $C$, which are very complicated and difficult to estimate in real situations, while $G$ is quite simple. An obvious inconvenience is, however, the fact that if $\gamma$ and $C$ are not small, some dynamic effects may arise as internal motions [17]. One simple way of suppressing the internal motion is to assign a simple nonlinear term that compensates $\gamma$ and $C$. Substituting (4) and $\frac{\partial}{\partial q}\left(J_{P} \dot{q}\right) \dot{q}=-J_{P} \ddot{q}$ into (3) we can derive a modified version of (4):

$$
\tau=J_{P}^{T}\left(f_{v}+M g\right)+\zeta(q, \dot{q})
$$

This yields the convergence (6) of GAF, provided $\zeta(q, \dot{q})$ is designed so that

$$
I(q) \ddot{q}+C(q, \dot{q})-\zeta(q, \dot{q})=0
$$

is stable. A conservative way to achieve this is simply to set $\zeta$ as a joint-wise damping term:

$$
\zeta=-D \dot{q}
$$

with a constant matrix $D>0$. Although we should investigate the structure of the left-hand side of (9), the controller (8) itself can be made very simple, as this example. See [18] and the related papers for the contact force control for redundant manipulators, where the authors analyzed the convergence of the internal motions with joint-wise damping and the position/force error of the end-effector.

Now we turn our eyes to multiple contact case. For simplicity, let us assume we are interested in total $\alpha$-contact points defined by $r_{S}=\left[r_{S 1}, r_{S 2}, \cdots, r_{S \alpha}\right]^{T} \in \mathcal{R}^{3 \alpha}$ and the associated contact forces $f_{S}=\left[f_{S 1}, f_{S 2}, \cdots, f_{S \alpha}\right]^{T} \in$ $\mathcal{R}^{3 \alpha}$ as shown in Fig. 2(b). See [11] for more general case. Of course, $r_{P}$ must lie within the supporting convex hull composed of $r_{S j}$. The relationship among GAF, CoP and the contact positions/forces can be written as

$$
\begin{gathered}
f_{P}=\sum_{j=1}^{\alpha} f_{S j} \\
x_{P}=\frac{\sum_{j=1}^{\alpha} x_{S j} f_{z S j}}{\sum_{j=1}^{\alpha} f_{z S j}} \quad, \quad y_{P}=\frac{\sum_{j=1}^{\alpha} y_{S j} f_{z S j}}{\sum_{j=1}^{\alpha} f_{z S j}},
\end{gathered}
$$

with $\bar{f}_{z S j}$ the normal contact forces, or in a simpler form

$$
f_{z P}\left[\begin{array}{c}
x_{P} \\
y_{P} \\
1
\end{array}\right]=\underbrace{\left[\begin{array}{cccc}
x_{S 1} & x_{S 2} & \cdots & x_{S \alpha} \\
y_{S 1} & y_{S 2} & \cdots & y_{S \alpha} \\
1 & 1 & \cdots & 1
\end{array}\right]}_{A_{z} \in \mathcal{R}^{3 \times \alpha}}\left[\begin{array}{c}
f_{z S 1} \\
f_{z S 2} \\
\cdots \\
f_{z S \alpha}
\end{array}\right]
$$

where $A_{z}$ represents a contact force distribution matrix. For some desired normal GAF $\bar{f}_{z P}$ and $\operatorname{CoP} \bar{r}_{P}$ we can calculate the corresponding desired contact forces. Specifically, we propose an optimal contact force distribution given by

$$
\left[\begin{array}{c}
\bar{f}_{z S 1} \\
\bar{f}_{z S 1} \\
\cdots \\
\bar{f}_{z S \alpha}
\end{array}\right]=A_{z}^{\#} \bar{f}_{z P}\left[\begin{array}{c}
\bar{x}_{P} \\
\bar{y}_{P} \\
1
\end{array}\right]
$$

with $A_{z}^{\#}=A_{z}^{T}\left(A_{z} A_{z}^{T}\right)^{-1}$. This solution is optimal in the sense that it minimizes the norm of the contact forces. Similarly we can distribute desired horizontal GAFs, $\bar{f}_{x P}$ and $\bar{f}_{y P}$, to the corresponding desired horizontal contact forces $\bar{f}_{x S j}$ and $\bar{f}_{y S j}$ through $A_{x}$ and $A_{y}$ (omitted).

Consequently, a desired GAF is distributed to desired contact forces by a simple matrix operation

$$
\bar{f}_{S}=A^{\#} \bar{f}_{P}\left[\begin{array}{c}
\bar{x}_{P} \\
\bar{y}_{P} \\
1
\end{array}\right],
$$

where $A$ is obtained by permutation of $A_{x}, A_{y}$ and $A_{z}$. Then, the commanded joint torques are obtained by

$$
\tau=J_{S}(q)^{T} \bar{f}_{S}+\zeta(q, \dot{q})
$$

where $J_{S}(q) \in \mathcal{R}^{3 \alpha \times n}$ represents the contact Jacobian from CoM to supporting contact points (derivative of $r_{S}$ by $q$ ). We always assume $\alpha \geq n \geq 3$, which is not at all restrictive However, of course $n \geq 3$ does not always imply the three components of GAF are manipulatable by the contact forces. In summary, we use (15) and (16) with $\bar{f}_{P}=f_{v}+M g$, instead of (8). It is important to note that the control torques generated by (16) are different from (8) because it utilizes the null space of $J_{P}$ by constraining the contact forces.

\section{Disturbance REJECTION CONTROL}

This section elaborates our disturbance rejection control shown in Fig. 1. This is composed of three components: balancing controller, stepping controller and the trigger.

\section{A. Balancing controller}

Let us define balancing control here is the asymptotical stabilization of the ground projection of CoM to some desired position in the supporting convex hull. One can employ a simple feed-forward and feedback law

$$
f_{u}=M \ddot{r}_{C}-K_{P C}\left(r_{C}-\bar{r}_{C}\right)-K_{D C}\left(\dot{r}_{C}-\dot{\bar{r}}_{C}\right)
$$

with the task-space PD-gains $K_{P C}, K_{D C}>0$.

Recall $\mathrm{CoP}^{2}$ can be written as

$$
x_{P}=\frac{z_{P} f_{x P}}{f_{z P}}, \quad y_{P}=\frac{z_{P} f_{y P}}{f_{z P}} .
$$

Therefore, for some given user force input $f_{u}$, the desired $\mathrm{CoP} \bar{r}_{P}$ is set by

$$
\bar{x}_{P}=\frac{\bar{z}_{P} f_{u x}}{m g+f_{u z}}, \quad \bar{y}_{P}=\frac{\bar{z}_{P} f_{u y}}{m g+f_{u z}}
$$

\footnotetext{
${ }^{2}$ Different from ZMP, CoP does not include the rate change of the angular momentum around $\mathrm{CoM}$. It represents the weighted sum of the translational ground contact forces. In this sense, definition of ZMP in [11][15] is different from this paper. Clearly, CoP cannot exit from the supporting region, but ZMP can.
} 
with $\bar{z}_{P}=-z_{C}$. Since the desired CoP should be limited to supporting convex hull, it in turn modifies the desired GAF. Finally, the modified GAF $\bar{f}_{P}$ as well as the desired CoP $\bar{r}_{P}$ are then used for determining the desired contact force closure in (15).

One of the practical issue for balancing control is the definition of the origin of $\Sigma_{W}$. Our simple solution is; defining the origin as the center of the feet contacting with the ground. ${ }^{3}$ This means the origin is always moving according to the current configuration of the robot. Gyro information is important.

Using the angular momentum around CoM is promising because we can apply large horizontal GAF regardless of ZMP [16]. However, such a rich full-body balancing scheme is a difficult optimal control problem with many constraints since it usually requires a large amount of joint velocities and the workspace, which are bounded. For a decoupled twolink planar model, the exact solution exists [19]. Inventing some practical and simple solution is our ongoing work. Currently we are using very simple attitude controller for the upper body. We are just superimposing a simple PD feedback control inputs, with a low gains, to the torso joint torques generated by (16), to maintain the upright posture.

\section{B. Stepping controller}

When the stepping motion is triggered, the swinging foot starts to track to a desired trajectory planned online to prevent the robot from falling. The trajectory was given by 3D Symmetric Walking Control (SWC) law with a fixed stride [11]. Let $r_{Q}=\left[x_{Q}, y_{Q}, z_{Q}\right]^{T} \in \mathcal{R}^{3}$ be the position vector from CoM to the center of the swinging foot. SWC constraints the motion of the swinging leg by

$$
\left[\begin{array}{l}
x_{P}+x_{Q} \\
y_{P}+y_{Q}
\end{array}\right]=0 .
$$

For some given $\bar{d}_{x y}$ (design parameter), the desired height of the swinging foot is given as a smooth curve of the stride

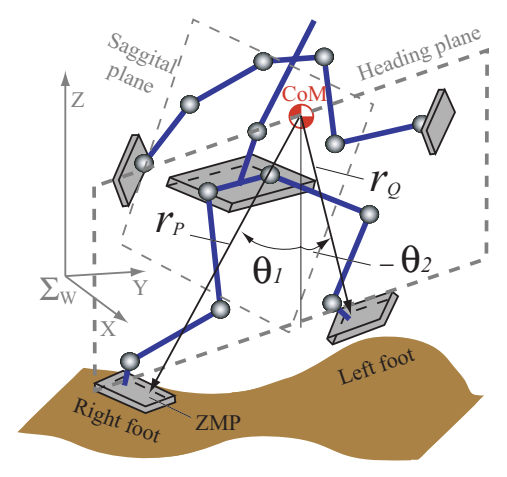

Fig. 3. Illustration of 3D symmetric walking: $r_{P}$ and $r_{Q}$ are the vector from CoM to ZMP and to the center of swinging foot respectively. $\theta_{1}$ and $\theta_{2}$ are the associated angle variables.

\footnotetext{
${ }^{3}$ Our previous description "Thanks to this simple solution, the controller does not have to know whether the foot is on or off the ground." (p.219 of [15]) was clearly wrong.
}

$d_{x y}:=\sqrt{\left(x_{Q}-x_{P}\right)^{2}+\left(y_{Q}-y_{P}\right)^{2}}$. It starts from zero, passing through a desired height, and terminating at zero when $\left|d_{x y}\right| \geq \bar{d}_{x y}$. Note that during walking the direction of the heading plane may changes, and the robot moves its foot to the direction of heading [13]. Therefore, SWC makes the projection of CoM locate at the center of supporting foot whenever the foot touches down. The mathematical properties of SWC presented in [13] are met in the same way by considering the angle variables $\theta_{1}$ and $\theta_{2}$ in Fig. 3. That is, the controlled walking gaits are not asymptotically stable, but Lyapunov stable symmetric orbits. Therefore, for every neighborhood of a walking gait, there exist a family of similar gaits with different energy level.

For disturbance rejection control, however, in addition to SWC we apply the balancing controller described in Section III-A all the time to achieve the asymptotical stability. From the passivity-based control viewpoint, the balancing controller can be regarded as a damping injection [14]. Note that the target $\mathrm{CoM}$ is always located at the center of the supporting region.

\section{Trigger to stepping motion}

We decided to choose the instance when the normal GRF of one foot becomes close to zero as the trigger to stepping action. The intuitive explanation of this transition rule is as follows. Initially the robot stands on both feet with its CoM located at the center of the supporting region. If the disturbance is applied, the robot tries to keep balance by controlling the CoM to the initial position. If too large disturbance is applied, CoM exits from the supporting region. However, due to our CoP feedback controller (17) and the contact force distribution (15), before CoM exit from the supporting region, the contact forces applied to the contact points which are located at the opposite side of the target position necessarily become zero. We choose this event as the trigger to stepping. More precisely, if the sum of the actual (not desired ones) vertical contact forces of one foot crosses a small threshold value, then the robot starts to make a step by that foot. Therefore, the transition is smooth and the ground reaction forces are continuous.

To note:

(1) If the robot is stiff, disturbances easily make either of the feet loose the contact forces. This is not the case for ours because of the passivity due to the full-body gravity compensation when equilibrium.

(2) Whether the desired contact forces becomes zero or not depends not only on the CoM states and the location of the contact points, but also the user force input $f_{u}$ in (17). For example, if $f_{u}=0$ (hence gravity compensation only), the contact forces becomes zero only if some joint angles have reached the limit.

(3) If we can control the angular momentum around CoM explicitly together with $\mathrm{CoP}$, the $\mathrm{CoP}$ can be restricted in some small range in the full supporting region, hence, the contact forces can be made positive all the time as long as physical constraints such as joint limits and maximum velocities/torques are not violated. 

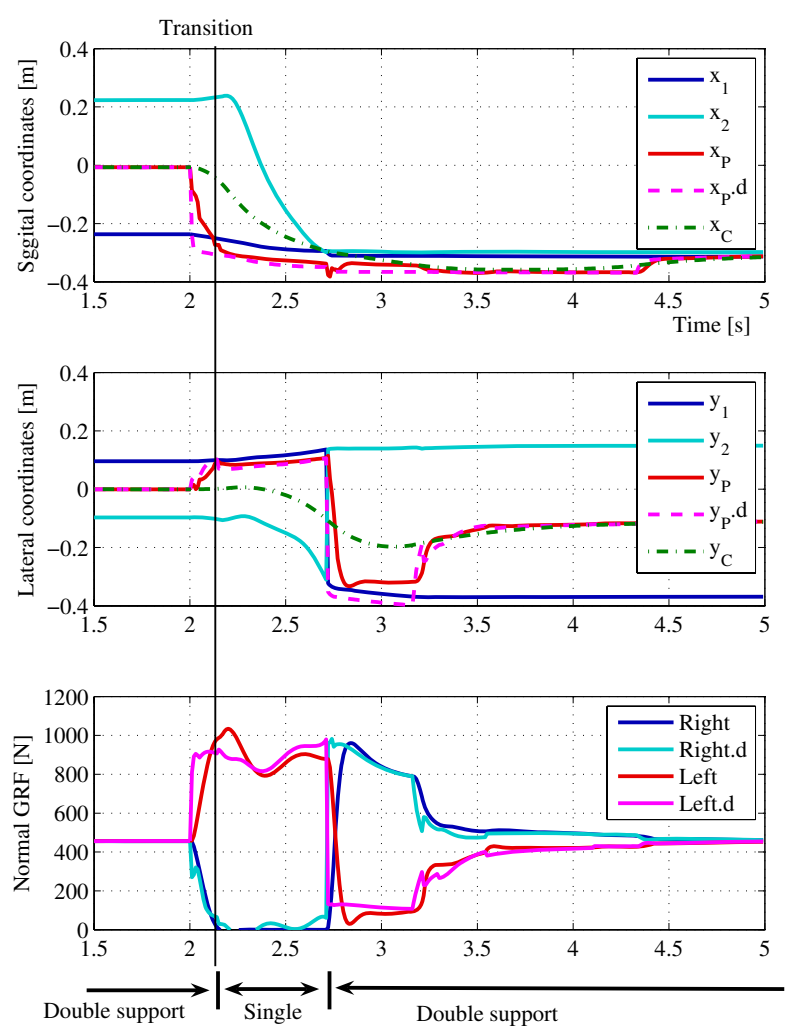

Fig. 4. Disturbance rejection simulation 1: the robot is suddenly pushed backward. The force is of the magnitude $550 \mathrm{~N}$ and applied to the abdomen at $2 \mathrm{~s}$ (the application time is $0.2 \mathrm{~s}$ ). The $\operatorname{CoP}\left(x_{P}, y_{P}\right)$ are shown with their desired values (indicated by '.d'). The center location of the supporting foot and the swinging foot are indicated by $\left(x_{1}, y_{1}\right)$ and $\left(x_{2}, y_{2}\right)$ respectively. $\left(x_{C}, y_{C}\right)$ represent the CoM.

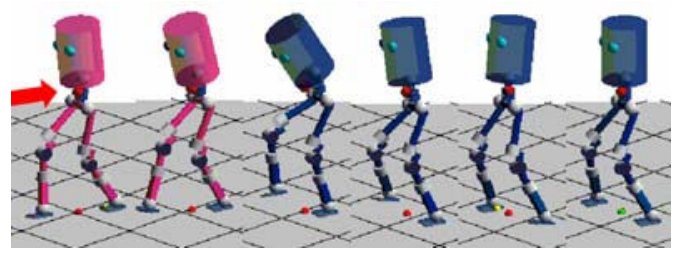

Fig. 5. Animation corresponding to Fig. 4: the robot takes one step to stop. Two red markers show CoM and its ground projection respectively, while the yellow and green ones indicate the desired $\mathrm{CoP}$ and the actual one respectively.

\section{Simulations}

This section shows some selected simulation results. Our control scheme is primitive and the results are premature in the sense that we did not consider the self collision between the limbs. Therefore, the applicable disturbances are very limited. We use a simplified simulation model without arms and head. The model is built on SD/FAST, where the ground contact is modeled as unilateral virtual springs and dampers. The hip height from the ground is $0.82 \mathrm{~m}$ at the upright posture, and the size of the sole is $0.15 \mathrm{~m} \mathrm{X} 0.1 \mathrm{~m}$. In the simulations the gains for (17) are set as $\left(K_{P C}, K_{D C}\right)=$ $(50 \times 93,5 \times 93)$ for the vertical motion and $\left(K_{P C}, K_{D C}\right)=$

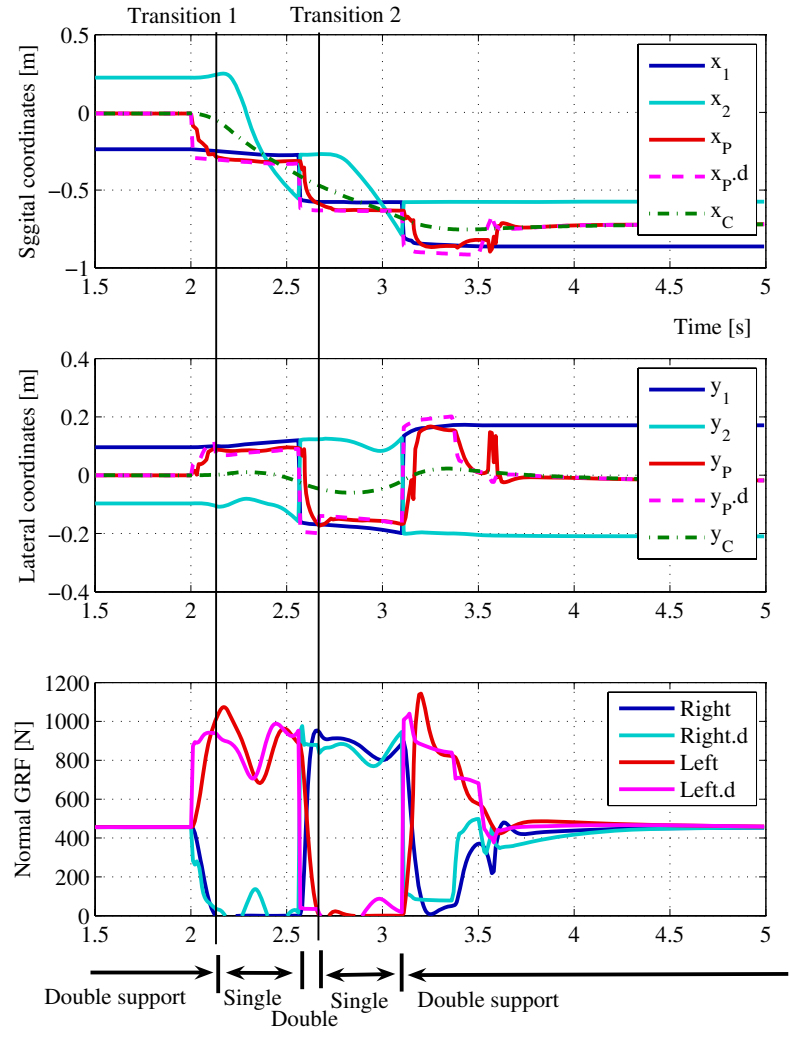

Fig. 6. Disturbance rejection simulation 2: the robot is suddenly pushed from front by 650 N. See Fig. 4 for the footnotes

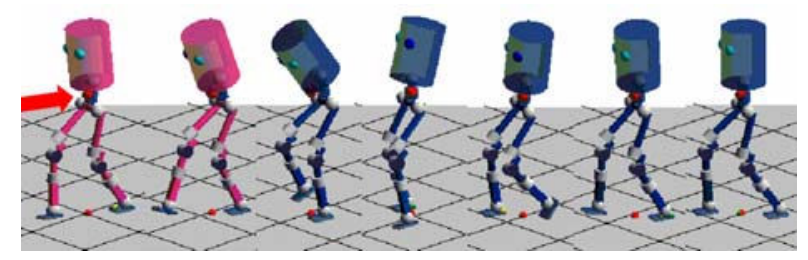

Fig. 7. Animation corresponding to Fig. 6: the robot takes two steps to stop.

$(100 \times 93,50 \times 93)$ for the horizontal motion, where 93 is the total mass. The desired CoM height set to $0.85 \mathrm{~m}$, while the horizontal positions are always set to the center of the support. The joint-wise damping in (10) has been set $D=\operatorname{diag}\left[d_{1}, d_{2}, \cdots, d_{n}\right]$ with $d_{\text {legs }}=2$ and $d_{\text {torso }}=8$. The feedback gains of the superimposed attitude control for upper body are all $10 \mathrm{Nm} / \mathrm{rad}$.

Fig. 4 and Fig. 5 show simulation results of the disturbance rejection with a large disturbance. The robot initially stands at the double support with its right leg put forward. Then a backward push of the magnitude $550 \mathrm{~N}$ is applied to the abdomen for $0.2 \mathrm{~s}$. Since the robot tries to keep the balance by the feedback controller (17), the desired CoP is repidly shift to the back left edge of the left sole as can be seen from the time profile of $x_{P} . d$ and $y_{P} . d$. However, since the external force is too large, the CoM $x_{C}$ rapidly moves backward. As a result, at $2.15 \mathrm{~s}(0.5 \mathrm{~s}$ earlier than the external 
push is released) the actual ground reaction force of the right leg crosses zero, and the event to stepping is triggered. After the single stepping motion, from $2.75 \mathrm{~s}$ the robot again tries to balance at the new supporting state. Finally, CoM converges to the center of the supporting region. Fig. 6 and Fig. 7 show the similar simulation result, but the magnitude of the disturbance is enlarged to $650 \mathrm{~N}$. This time the robot takes two steps to completely stop.

Note that in both simulations there can be found errors between the actual GRF and the desired GRF (=-GAF), hence, between the actual ZMP and the desired one too. The errors come from the uncompensated nonlinear dynamics and not using of the desired feed-forwad temrs in (17). At steady state they coincide with each other. Note again that we are using the measured GRF only for the decision making of the transition, but not for the balancing controller.

\section{EXPERIMENTS}

\section{A. Humanoid platform and the joint torque control}

The humanoid platform shown in Fig. 1 is developed by SARCOS (www.sarcos.com). The robot is called CB (Computational Brain). The legs and arms have 7 DOF, 3 DOF on the neck, and the torso has 3 DOF. There are total 50 DOFs including the fingers (12 DOF) and the eye cameras (4 DOF). Most of the DOFs are driven by hydraulic servo actuators. The total mass is $93 \mathrm{~kg}$ when this paper is published. The robot is installed with position sensors, force sensors and attitude sensors. The actuators are controlled by small-sized digital controllers supporting position, velocity and force feedback, which are inter-connected by high-speed intra network. See [20] for the details.

The joint torque control is solely based on the force feedback of the force sensors attached to each joint. However, the joints, actuators and sensors are not collocated in our robot. Therefore we implemented a joint-wise force-torque transformation based on the individual joint kinematics. Once having the joint torque calibration, it is straightforward to apply the controller. Actually, the control procedure summarized in Section II is very simple and can be executed with a low computational cost. So far, the torque control performance of our humanoid platform is found to be steady and repeatable. However, there are force tracking delays. The delays can be minimized by maximizing the force gains in the local joint controllers, but high gains cause undesirable resonance. Fortunately the tracking delays have the similar effect of joint-wise damping, this can be effectively utilized for suppressing the internal motions as described in (10). In other words, we can change the damping by simply tuning the force gains.

\section{B. Push recovery from unknown external forces without triggering the stepping}

Fig. 8 shows the typical performance of our balancing controller when a side force is applied. Needless to say, the robot is neither supported by the hydraulic hoses and cables. This result nicely shows the relationship between the CoM error and the ground reaction force distribution. As in this

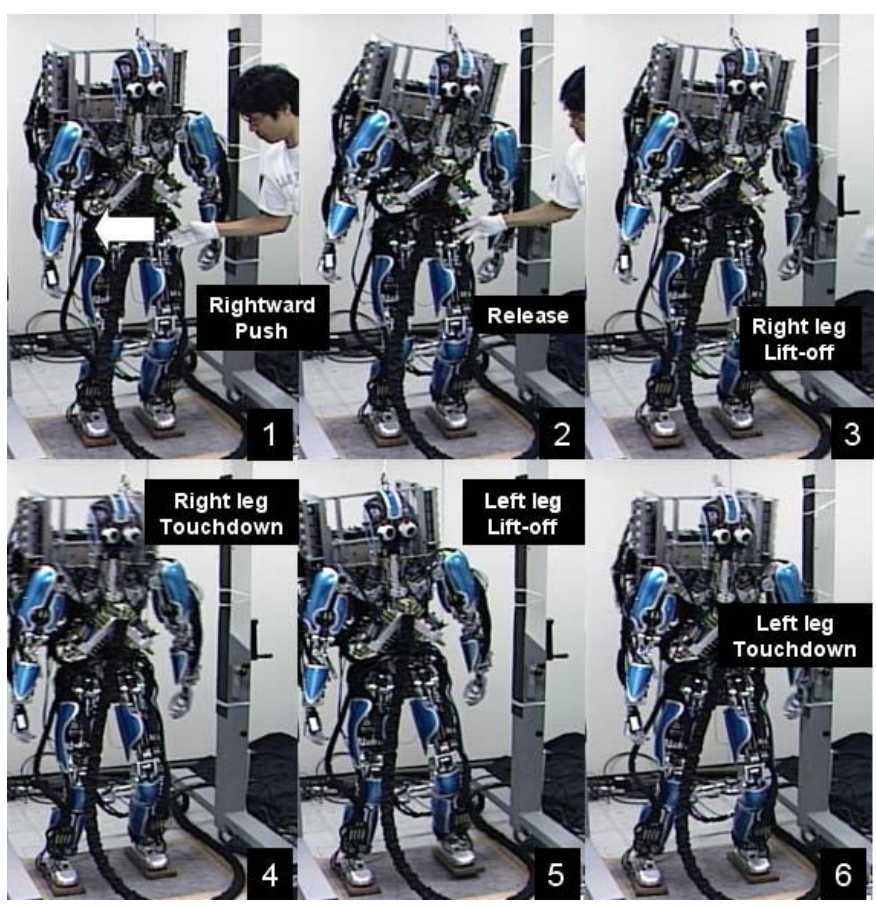

Fig. 8. Our force distribution law makes some of the contact forces small when a large error in CoM is detected. As a result, a foot may lift off the ground.
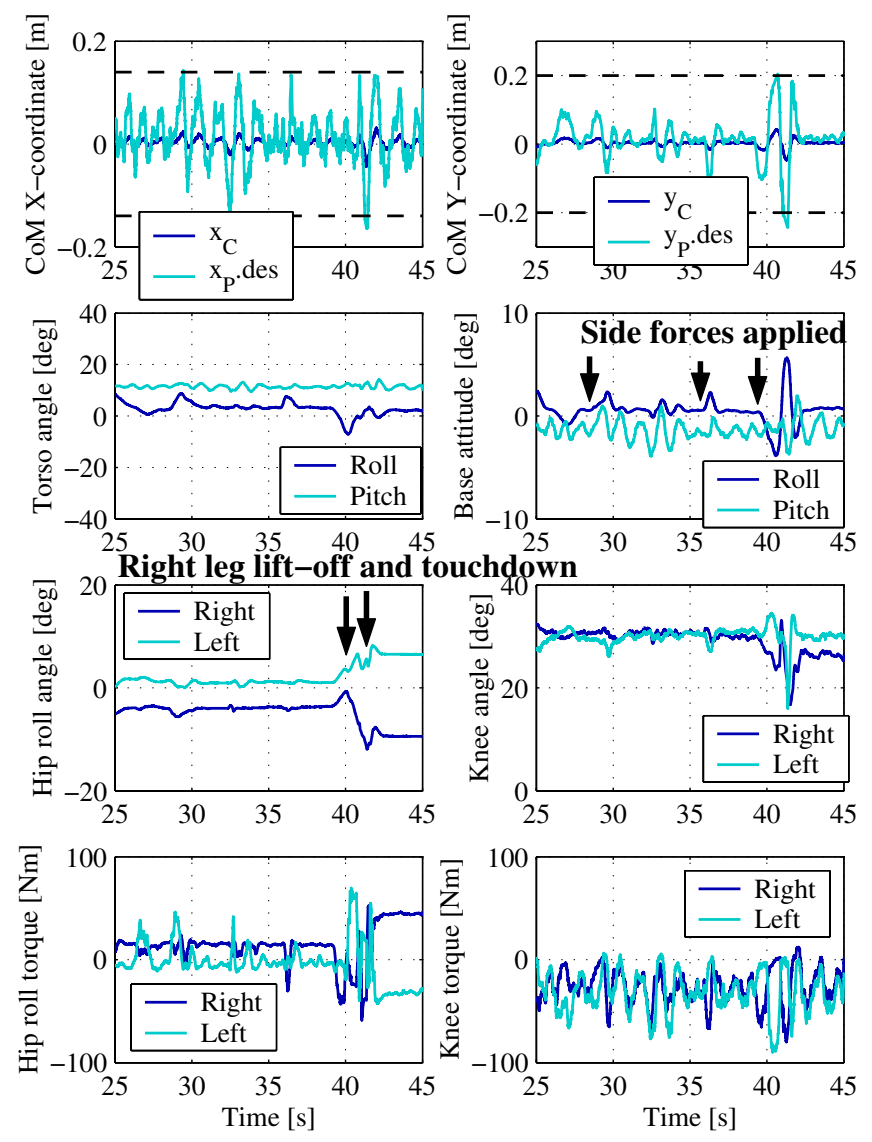

Fig. 9. Experimental data corresponding to Fig. 8. 


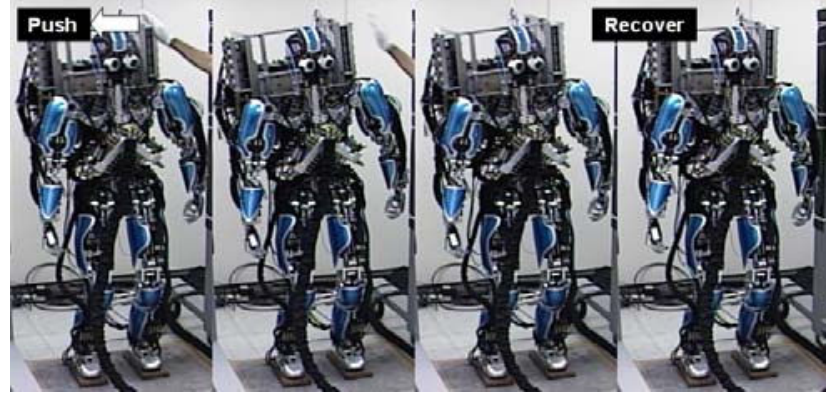

Fig. 10. Recovery from the side push at the back

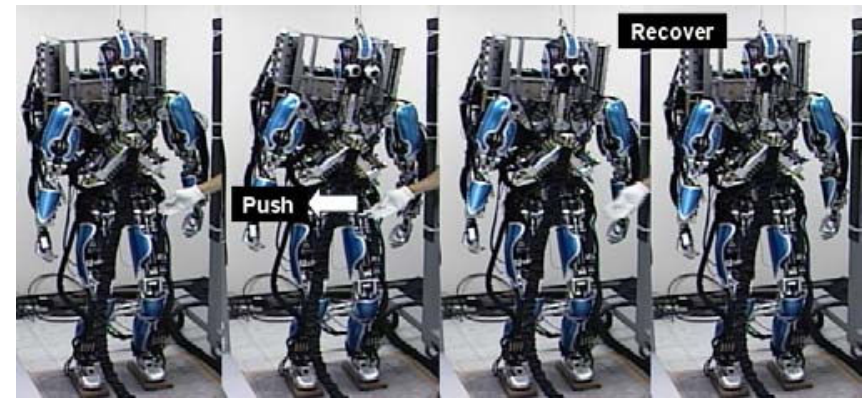

Fig. 11. Recovery from the side push at the hip

case, CoM is perturbed from the desired equilibrium (zero), and the desired normal contact forces become close to zero when the ground projection of $\mathrm{CoM}$ and $\mathrm{CoP}$ is far located each other. Because of this, it happened one foot lifted off the ground. In this example, however, we did not triggered the stepping controller. Therefore, the lifted foot moves purely as a result of the balancing control. At the same time the CoM moves back to the center of the feet. Then the lifted foot touches the ground. This process repeats once more, and finally the robot balances, but with a different configuration from the initial one. Fig. 9 shows the time evolusion of this experimet. The top two graphs show the position of CoM and desired CoP. The limitations of the desired CoP in Xdirection are indicated by two dashed lines, $\pm 0.15 \mathrm{~m}$.

Note that we do not specify which joints should move in compliance with the disturbances, for example, we do not use any weighting matrix as in some inverse kinematics approach. The weighting is automatically done by the Jacobian transpose (16) (not inverse), and the compensating contact forces are distributed to the joint torques of whole body. Therefore, if we push the robot torso, the torso compliantly follows first, and then the lower body begins to compensate for the CoM error. Similarly, if we push the hip, the hip moves first. This can be seen from Fig. 10 and Fig. 11. Although the controller does not measure the external force, the responce to the external force is immediate. This is because most of the joints are commanded zero torque when the robot has upright posture (equilibrium).

\section{Disturbance rejection with stepping}

Fig. 12 and 13 show a preliminary result on the disturbance rejection experiment. The robot is allowed to transit from the double support phase to the single according to the transition rule made in Section III-C, based on the measured GRF. Different from the simulation result, there can be seen a chattering in the GRF of the to-be-lifted leg just after the trigger as indicated by a dashed circle in the bottom graph. This is because our stepping controller is too sensitive to the current positions and velocities of the supporting foot and the CoM. Reducing the sensitivity is left for the future work.

\section{SUMmary}

This paper proposed a simple passivity-based disturbance rejection scheme for force-controllable biped humanoid robots. It was composed of three sub-controllers: 1) a balancing controller; 2) a stepping controller; and 3) the trigger. Numerical simulations and experiments evaluated the effectiveness of the proposed method. Although the method is incomplete in the sense that the self-collision between the limbs is ignored, preliminary experimental results demonstrated that the proposed method could actually maintained balance even under unknown large external perturbations. Technical issues on the joint torque control were also presented. Our future work includes extension to CoP-free balancer, joint torque minimization, and joint angle limitation

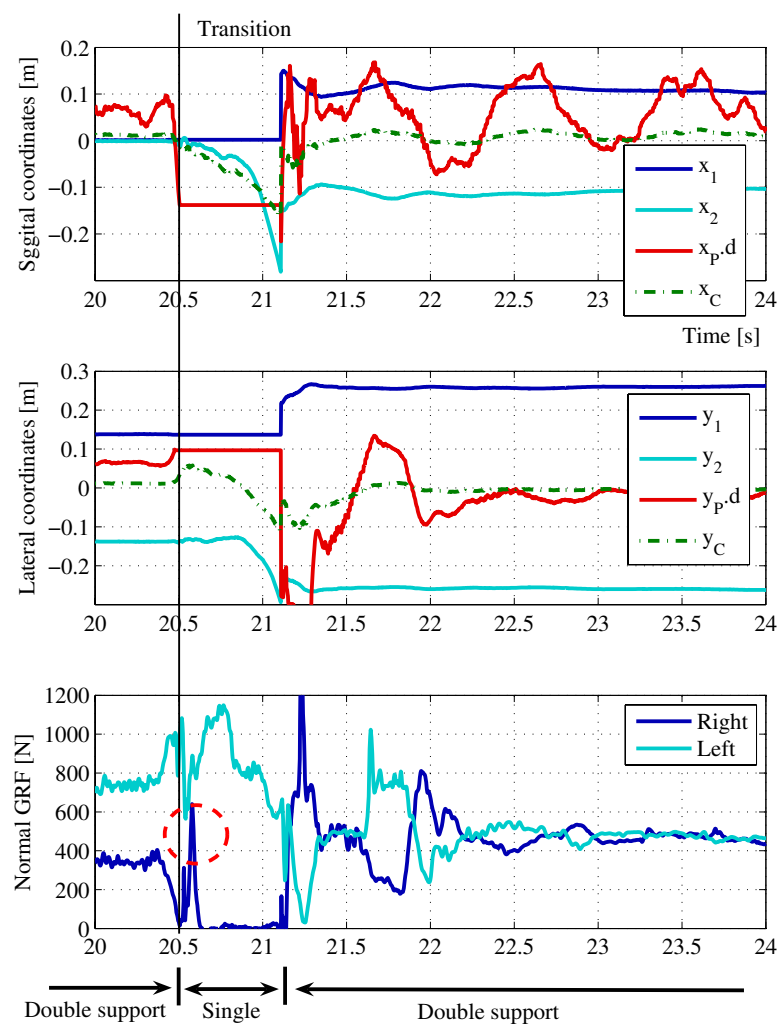

Fig. 12. Disturbance rejection experiment: The top two graphs show the loci of CoM and the foot positions $\left(x_{1}\right.$ and $x_{2}$ are the $X$-position of the right foot and left foot respectively.). After the touchdown the robot again balances at wider stance. There is a chattering in GRF indicated by a dashed circle in the bottom graph. The desired $\mathrm{CoP}$ is oscillating in forward direction by $0.1 \mathrm{~m} \mathrm{p}$-t-p, but the error of CoM is small. 


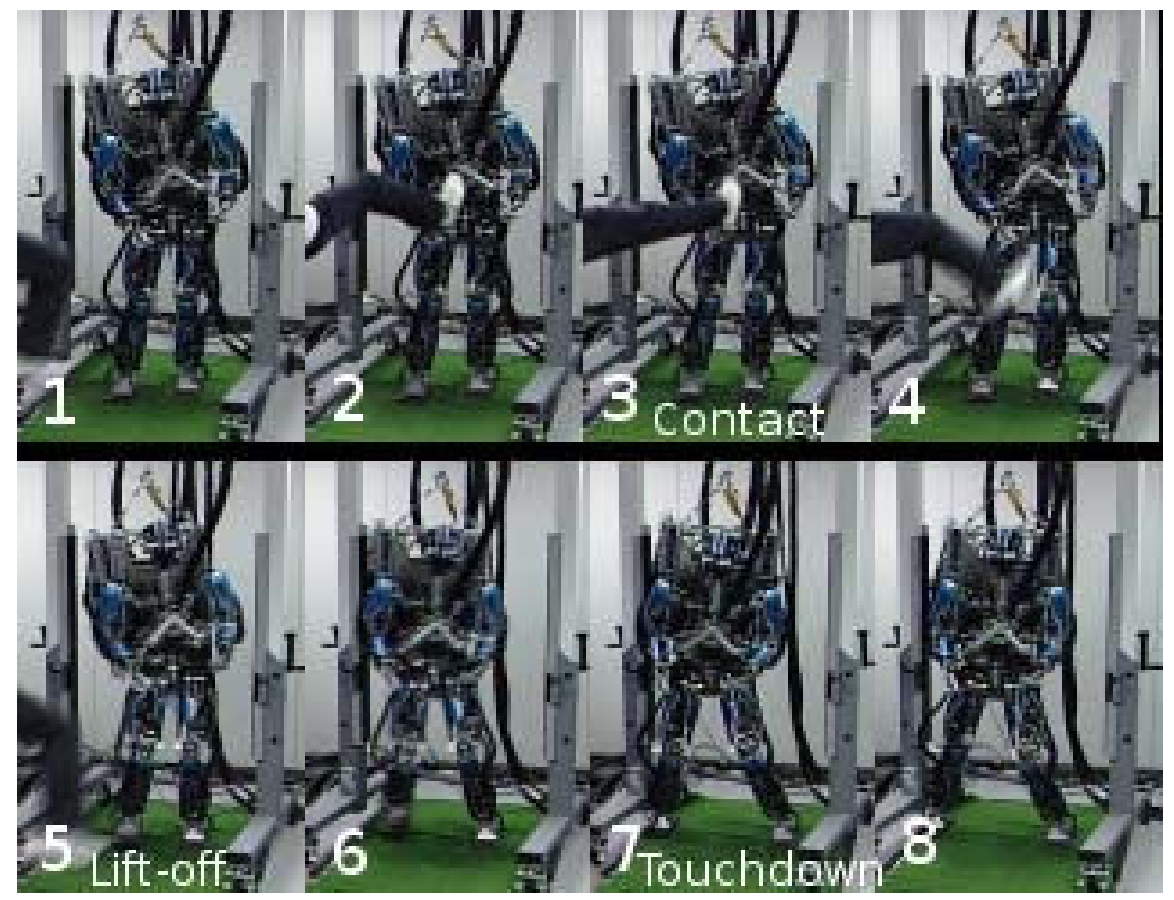

Fig. 13. Snap shots of the disturbance rejection experiment: A large amount of backward push is applied to the robot chest. The robot steps back to keep the balance. Due to the lack of the space the swinging (right) foot hits the side of the gantry.

as well as self-collision avoidance.

\section{ACKNOWLEDGEMENT}

The authors would like to thank the support of SARCOS Inc. and the Keihanna branch of the National Institute of Communication Telecommunication (NiCT), Japan. The authors gratefully acknowledge the contribution of J. Hart (ATR) and N. Nakano (ATR) for the experimental setups.

\section{REFERENCES}

[1] A. Takanishi, T. Takeya, H. Karaki, and I. Kato, "A control method for dynamic biped walking under unknown external force," in Proc. of IEEE International Workshop on Intelligent Robots and Systems, July 1990, pp. 795-801.

[2] K. Hirai, M. Hirose, Y. Haikawa, and T. Takenaka, "The development of the honda humanoid robot," in Proc. of IEEE International Conference on Robotics and Automation, Leuven, Belgium, May 1998, pp. $1321-1328$.

[3] J. Yamaguchi, E. Soga, S. Inoue, and A. Takanishi, "Development of a bipedal humanoid robot-control method of whole body cooperative dynamic biped walking," in Proc. of IEEE International Conference on Robotics and Automation, May 1999, pp. 368 - 374.

[4] S. Kajita, F. Kanehiro, K. Kaneko, K. Fujiwara, K. Harada, K. Yokoi, and H. Hirukawa, "Resolved momentum control: humanoid motion planning based on the linear and angular momentum," in Proc. of IEEE/RSJ International Conference on Intelligent Robots and Systems, Las Vegas, USA, 2003, pp. 1644-1650.

[5] _ - "Biped walking pattern generation by using preview control of zero-moment point," in Proc. of IEEE International Conference on Robotics and Automation, vol. 2, Taipei, Taiwan, Sep. 2003, pp. $1620-1626$.

[6] S. A. Setiawan, S. Hyon, J. Yamaguchi, and A. Takanishi, "Physical interaction between human and a bipedal humanoid robot -realization of human-follow walking-," in Proc. of IEEE International Conference on Robotics and Automation, Detroit, May 1999, pp. 361-367.

[7] J. Pratt, C.-M. Chew, A. Torres, P. Dilworth, and G. Pratt, "Virtual model control: An intuitive approach for bipedal locomotion," International Journal of Robotics Research, vol. 20, no. 2, pp. 129-143, 2001.
[8] L. Sentis and O. Khatib, "Synthesis of whole-body behaviors through hierarchical control of behavioral primitives," International Journal of Humanoid Robotics, 2005.

[9] J. Park and O. Khatib, "A whole-body control framework for humanoids operating in human environments," in Proc. of IEEE International Conference on Robotics and Automation, Orlando, USA, 2006, pp. 1963-1969.

[10] O. Khatib, "A unified approach for motion and force control of robot manipulators: The operational space formulation," IEEE Journal of Robotics and Automation, vol. RA-3, no. 1, pp. 43-53, 1987.

[11] S. Hyon and G. Cheng, "Passivity-based full-body force control for humanoids and application to dynamic balancing and locomotion," in Proc. of IEEE/RSJ International Conference on Intelligent Robots and Systems, Beijing, China, Oct. 2006, pp. 4915-4922.

[12] S. Hyon, "Hamiltonian-based running control of dynamic legged robots," Systems, Control and Information, vol. 49, no. 7, pp. 260265, 2005 (in Japanese).

[13] S. Hyon and T. Emura, "Symmetric walking control: Invariance and global stability," in Proc. of IEEE International Conference on Robotics and Automation, Barcelona, Spain, Apr 2005, pp. 1455-1462.

[14] A. J. van der Schaft, L2-Gain and Passivity Techniques in Nonlinear Control. Springer, 1999.

[15] S. Hyon and G. Cheng, "Gravity compensation and full-body force interaction for humanoid robots," in Proc. of IEEE-RAS International Conference on Humanoid Robots, Genova, Itary, 2006, pp. 214-221.

[16] S. Hyon, N. Yokoyama, and T. Emura, "Back handspring of a multilink gymnastic robot - reference model approach," Advanced Robotics, vol. 20, no. 1, pp. pp.93-113, 2006.

[17] R. M. Murray, Z. Li, and S. S. Sastry, A Mathematical Introduction to Robotic Manipulation. CRC Press, 1994.

[18] S. Arimoto, H. Hashiguchi, and R. Ozawa, "A simple control method coping with a kinematically ill-posed inverse problem of redundant robots: Analysis in case of a handwriting robot," Asian Journal of Control, vol. 7, no. 2, pp. 112-123, 2005.

[19] J. Pratt, J. Carff, S. Drakunov, and A. Goswami, "Capture point: A step toward humanoid push recovery," in IEEE-RAS International Conference on Humanoid Robots, Genova, Itary, 2006, pp. 200-207.

[20] G. Cheng, S. Hyon, J. Morimoto, A. Ude, and S. C. Jacobsen, "CB A humanoid research platform for exploring neuroscience," in Proc. of IEEE-RAS International Conference on Humanoid Robots, Genova, Itary, 2006, pp. 182-187. 